\title{
Just like new: Newly segregated old objects capture attention
}

\author{
SHAWN E. CHRIST and RICHARD A. ABRAMS \\ Washington University, St. Louis, Missouri
}

\begin{abstract}
Subjects searched for target letters that were either contained in or segregated from a group of distracting letters and were either moving or stationary. Subjects were faster to identify targets that had recently become segregated from the group regardless of whether the segregation was accomplished by (1) the element itself moving away from the group or (2) the group moving away from the segregated element. The results show that attention can be captured by newly apparent objects that are produced by a new grouping of an existing scene.
\end{abstract}

In navigating our environment, we are faced with a steady stream of perceptual objects entering and exiting our visual field. As a result, we must determine which objects are to receive attentional priority and be processed first. An observer's goals play an important role in this process. For example, a person may choose to search for their green coupe in a parking lot filled with a variety of cars, and, as a result, green coupes might be assigned a high priority. This type of attentional selection is referred to as goal directed.

Importantly, the priority with which we select objects in our environment can also be affected by bottom-up, automatic processes that are driven by low-level stimulus properties or events. For example, the black-and-white sedan with the flashing lights and pulsating siren may attract our attention despite the fact that it might not be the sought-for color or style. In contrast to goal-directed attention, this stimulus-driven attentional capture occurs largely independent of the viewer's intentions (for a review, see Rauschenberger, 2003a).

One event that has been shown to capture attention in a stimulus-driven manner is the appearance of a new perceptual object. This conclusion has been reached in part through the use of visual search tasks in which subjects must search for a target stimulus among distractors. Researchers have found that when the target is presented in an object that is new in the display, subjects are quicker to respond than when the target appears in a previously present "old" object (e.g., Samuel \& Weiner, 2001; Yantis $\&$ Jonides, 1984). Moreover, the amount of time needed to identify such new targets does not increase as a function of the search set size, suggesting that the object receives attentional priority and is inspected first.

The work reported here was supported by Grant BCS-0079594 from the National Science Foundation. Correspondence should be addressed to S. Christ, Department of Psychological Sciences, 210 McAlester Hall, University of Missouri, Columbia, MO 65211 (e-mail: research@ shawnchrist.com).
Most of the early studies exploring capture by new objects used abrupt visual onsets involving changes in luminance to define the new objects (e.g., Yantis \& Jonides, 1984). This leaves open the possibility that it was the luminance change and not the appearance of a new object that was responsible for capturing attention. More recently, however, similar results have been obtained using discontinuities in display features other than luminance (e.g., texture differences) to define the objects (Gellatly, Cole, \& Blurton, 1999; Rauschenberger \& Yantis, 2001; Yantis \& Hillstrom, 1994; see also Oonk \& Abrams, 1998).

In an important study, Hillstrom and Yantis (1994) also examined capture without introducing any new elements into the scene at all. Hillstrom and Yantis studied what would occur when a preexisting element, previously part of a larger perceptual grouping, suddenly segregated itself from the original group. In their study, one letter in a set of letters became segregated by moving away from the others, which remained stationary. If new objects truly capture attention, then a newly segregated item might attract attention in much the same way that a newly presented item does. Indeed, Hillstrom and Yantis found that such newly segregated items did capture attention. (Their conclusion was based on the fact that the segregation increased the effect of the letter's compatibility in a local/global judgment.) Other experiments in their study allowed Hillstrom and Yantis to rule out capture by the motion of the element itself - instead, they concluded that motion might create a new object, as it had in their display, and the new object, in turn, is what can capture attention. Taken together, these findings support the conclusion that new objects and newly perceived objects attract attention even in the absence of transients and other display changes that often accompany their appearance.

Recently, we investigated the role of motion and motion transients in attentional selection (Abrams \& Christ, 2003, 2004a, 2004b, 2005). In our experiments, subjects searched for target letters among moving and stationary objects that had undergone several different types of motion transitions. We found that movement per se did not 
capture attention, but the onset of movement did capture attention. In light of that finding, an alternative explanation for Hillstrom and Yantis's (1994) results is possible: Given that the segregation of the soon-to-be-new element in their experiments was always accompanied by a motion onset, it is possible that attention was captured by the motion onset and not by the newly segregated object. In fact, it seems possible that either the motion onset or the new perceptual grouping alone would each have been sufficient to attract attention in Hillstrom and Yantis's experiments. Whereas motion onset alone has been shown to capture attention (Abrams \& Christ, 2003, 2004a, 2004b, 2005), the possibility of capture generated solely on the basis of a new grouping remains untested, and this was the focus of the present experiments.

The present study was designed to determine if a newly segregated preexisting object, in the absence of a motion onset (or any other transient event known to capture attention), is capable of attracting attention. If it is, that would further bolster theories that stress the priority given to new objects. In our first experiment, we attempted to replicate the Hillstrom and Yantis (1994) result in a modified visual search paradigm with new stimuli. Then, we used the new stimuli to examine attentional capture by newly segregated objects in the absence of motion onset.

\section{EXPERIMENT 1}

The present experiment was designed to replicate the general pattern of results reported by Hillstrom and Yantis (1994) using a visual search paradigm (as opposed to a local/global letter judgment task). For this purpose, we presented an initial display consisting of five figure-eight placeholders in close spatial proximity to each other, thus increasing the likelihood that they would be perceptually grouped together. After a few seconds, one of the placeholders began to move while the others remained stationary. Concurrent with this event, all of the placeholders were replaced by letters, one of which was the target.

Although each of the items were equally likely to be the target, if attention were attracted by the newly segregated item, then the subjects should be faster to identify the target letter if it occurred in this item relative to the other items.

\section{Method}

Subjects. Ten undergraduates participated in the experiment. All were experimentally naive and received course credit in return for their efforts.

Apparatus and Procedure. The subjects were seated in front of a video display in a dimly lit room, and they were encouraged to maintain fixation at the center of the display throughout the trial. The sequence of events on each trial is shown in Figure 1. At the beginning of each trial, a vertically aligned row of five placeholder stimuli was presented at the center of the display. Each placeholder was an " 8 " that was $1^{\circ}$ high and $0.5^{\circ}$ wide. The placeholders were equally spaced, with a $0.25^{\circ}$ gap between adjacent placeholders.

Following a delay that varied randomly between 1,667 and $5,000 \mathrm{msec}$, one of the placeholders (excluding the top and bottom ones) began to move toward the right side of the display. ${ }^{1}$ The move- ment was accomplished by displacing the relevant item at a rate of 1 pixel per video display refresh $(16.7 \mathrm{msec})$. The resulting speed was approximately $3 \%$ sec.

The onset of motion coincided with the presentation of the search display. At that time, segments from each placeholder were removed to reveal letters. One of the placeholders became the letter "S" or "H," representing the target stimulus. All remaining placeholders were replaced by distractor letters (either all "E"s or all "U"s). The subjects were instructed to respond to the target's identity as quickly as possible by pressing one of two keys (" $z$ " or "/" key) on the keyboard.

The search array remained visible until the subject responded or $3,000 \mathrm{msec}$ had elapsed. If the subject responded incorrectly, a brief tone followed by the message "Wrong Response" was presented. A tone and relevant message (i.e., "Too Early" or "Too Slow") was presented if a subject responded less than $300 \mathrm{msec}$ after array onset or failed to respond within $3,000 \mathrm{msec}$, respectively. The intertrial interval was $1,000 \mathrm{msec}$.

Design. Following 20 practice trials, the subjects served in 240 experimental trials. Trial presentation was balanced such that the target was equally likely to appear in each of the five items. Thus, on one fifth of the trials, the target appeared in the item that moved away from the group; on the remaining trials, it appeared in one of the items included in the original grouping. Furthermore, the target was equally likely to be an "S" or "H," and the distractor letters were equally likely to be "E" or "U" for each type of target. The targetto-response key mapping was counterbalanced across subjects. Trial types were randomly mixed. At intervals of 40 trials, the subjects were given the opportunity to take a break.

\section{Results and Discussion}

Mean reaction times (RTs) are shown in the left pair of bars in Figure 2 separately for trials on which the target appeared in the newly segregated item and in an item in the original grouping. The subjects were faster to identify the target when it appeared in a newly segregated item $(M=621.6 \mathrm{msec}, S D=93.0)$ relative to the original grouping $(M=712.1 \mathrm{msec}, S D=126.0)[t(9)=6.94$, $p<.001]$. Error rates were low and did not depend on the condition (newly segregated item, $M=3.5 \%$; original grouping, $M=3.7 \%$ ).

Thus, using a visual search paradigm, we successfully replicated the pattern of results reported by Hillstrom and Yantis (1994): We found an attentional advantage for an item that had recently become segregated from a perceptual group on the basis of motion.

\section{EXPERIMENT 2}

In Experiment 1, as in the Hillstrom and Yantis (1994) study, an object became segregated from a perceptual group because it moved. The event captured attention either because some aspect of the movement captured attention or because the newly apparent perceptual object captured attention, or both. In our recent work, we have shown that movement per se is not sufficient to capture attention, but movement onset is sufficient (Abrams \& Christ, 2003, 2005). Thus, it seems likely that the onset of movement in the present Experiment 1 and in Experiments 2 and 3 of the Hillstrom and Yantis (1994) study would have been sufficient to attract attention. What is presently not known, however, is whether a newly apparent perceptual object is sufficient to capture attention. In 


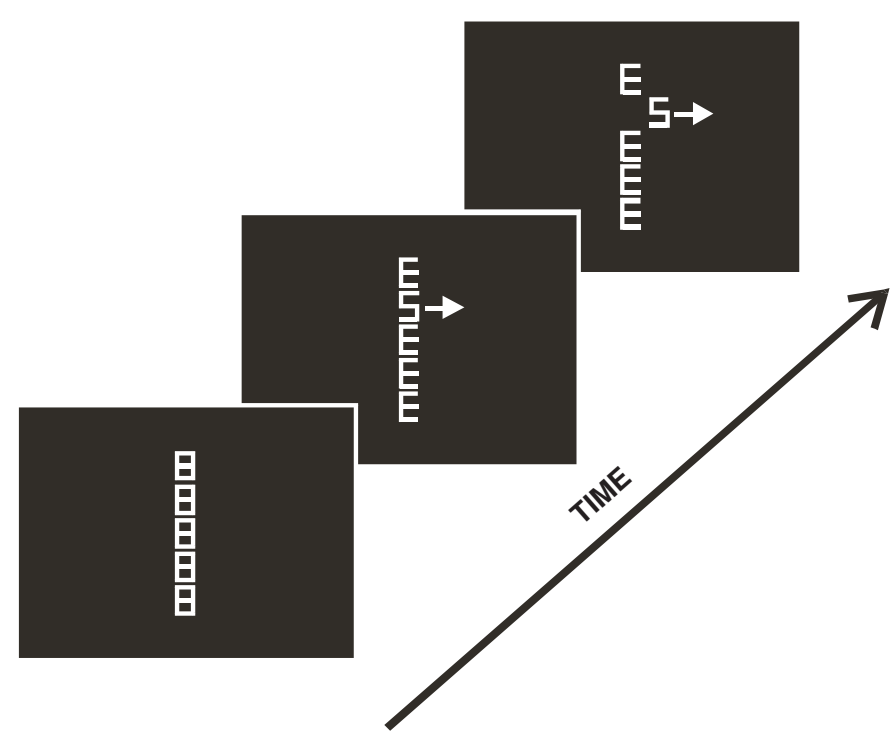

Figure 1. Sequence of events on a trial in Experiment 1. The arrow indicates motion of one of the elements, but the arrow did not appear in the display. Motion onset was coincident with the appearance of the search array (i.e., the letters in the display).

the present Experiment 1 and in the Hillstrom and Yantis study, it was not possible to separate the effects of segregation from the effects of motion onset. Our goal in the present experiment was to answer this question under conditions in which the segregation occurred without motion onset of the element in question.

To accomplish that, we used two new types of motion events to segregate an object from a group. In one condi- tion, an object became segregated from a moving group by having the group continue to move while the object ceased moving; in the other, an object was segregated from a moving group by continuing to move itself while the other elements in the group ceased moving. Neither of these conditions contained motion that would itself be expected to attract attention, yet both contained an object that became segregated from a group. If the newly appar-

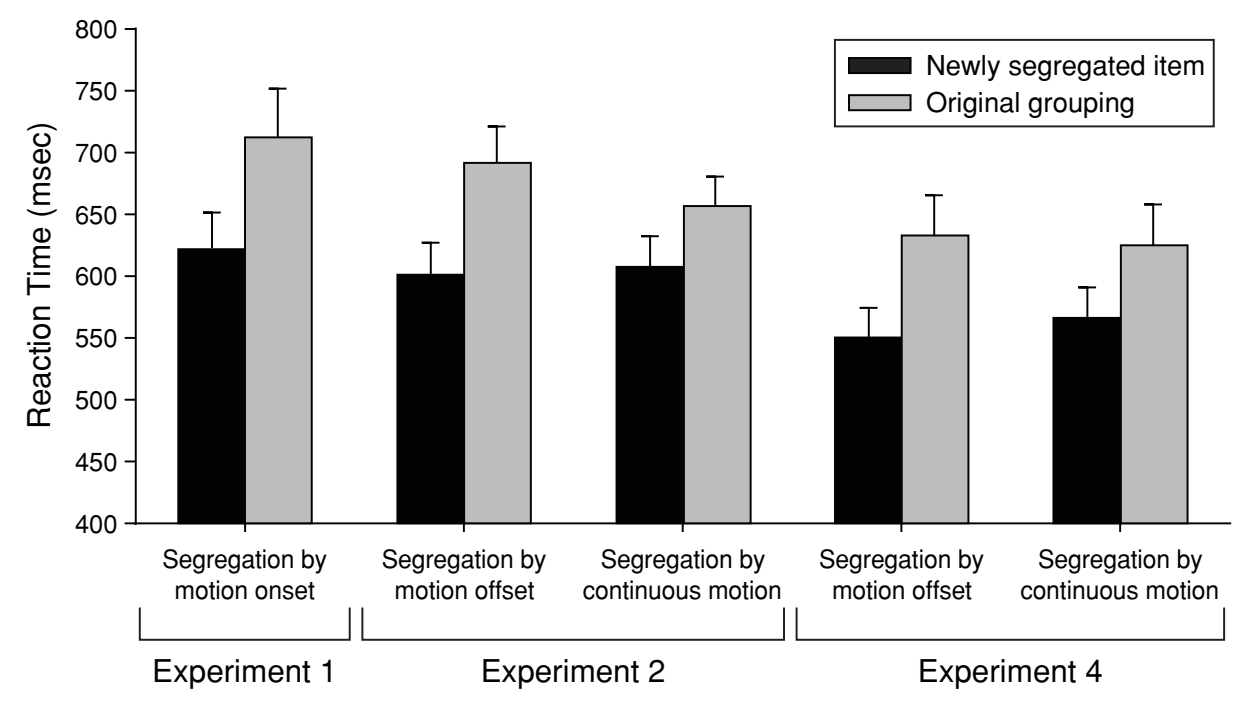

Figure 2. Mean reaction times for target identification in Experiments 1, 2, and 4, shown separately for each display type (segregation by motion onset, by motion offset, or by continuous motion) and each target type (newly segregated item or original grouping). Error bars show the standard error of the mean. 
ent object is sufficient to attract attention, then it should enjoy a benefit to target identification even under those circumstances.

\begin{abstract}
Method
Subjects. Ten students drawn from the same population as studied earlier participated. None had served previously.

Apparatus, Procedure, and Design. This experiment was similar to Experiment 1, with differences noted here. A grouping of five vertically aligned placeholders was presented initially $10^{\circ}$ to the left of the center of the display. The grouping immediately began moving to the right at a speed of $3^{\circ} \mathrm{sec}$. Following a random delay of 1,667-5,000 msec, when the group was within $5^{\circ}$ of the center of the display, a movement transition occurred: On half of the trials, one of the placeholders (excluding the top and bottom ones) stopped moving while the remaining placeholders continued to move across the display. The unique element in this type of display was thus segregated by motion offset (segregation by motion offset display; Figure 3A). On the other half of the trials, all but one of the placeholders stopped, and the remaining placeholder continued its motion (segregation by continuous motion display; Figure 3B).

As in Experiment 1, the placeholders were changed to reveal the search display coincident with the movement transition. The target was equally likely to appear in each of the five items. The subjects were instructed to identify the target as quickly as possible.

Following 20 practice trials, the subjects served in 360 experimental trials. Trial types were randomly mixed.
\end{abstract}

\section{Results and Discussion}

Mean RTs from each condition are shown in the middle pairs of bars in Figure 2. The data were analyzed using a 2 (target; newly segregated item vs. original grouping) $\times 2$ (display; segregation by motion offset vs. segregation by continuous motion) ANOVA. Most important, there was a main effect of target, with the subjects faster to respond when the target appeared in the newly segregated item than when it appeared in the original grouping $[F(1,9)=$ $71.71, p<.001]$. Post hoc analyses confirmed that this was the case regardless of whether the group moved away from the segregated item (segregation by motion offset display) $[t(9)=7.41, p<.001]$ or the item moved away from the group (segregation by continuous motion display) $[t(9)=7.77, p<.001]$.

Error rates were less than $4 \%$ in all conditions and did not depend on target or display type, nor was there an interaction $[F \mathrm{~s}(1,9)<1.3]$.

Our results demonstrate attentional capture caused by the segregation of an object from a preexisting group under conditions in which the motion of the object itself could not have caused the capture. Thus, these findings are consistent with the notion that the newly segregated item is treated like a new perceptual object and, as such, receives attentional priority.

Although not central to our primary goal, it is worth noting that we also found a main effect of display type $[F(1,9)=6.78, p<.05]$ and an interaction between target and display type $[F(1,9)=15.58, p<.005]$. As can be seen in Figure 2, both of these effects appear to have been driven primarily by RT differences in the original grouping condition across the two display types. Post hoc analyses showed that the subjects were slower to respond
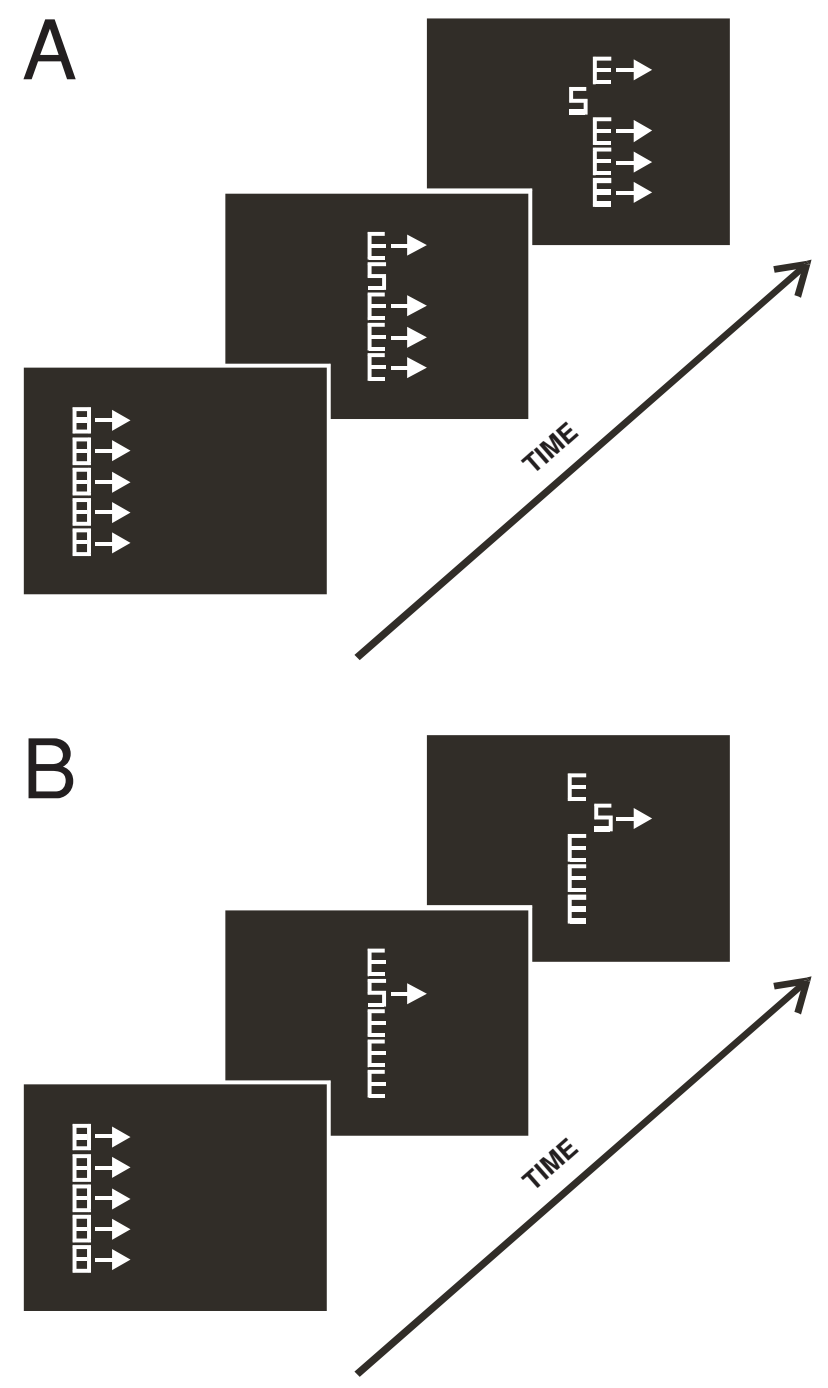

Figure 3. Sequence of events on a trial in Experiment 2. The two different display types - segregation by motion offset $(A)$ and segregation by continuous motion (B) - are shown separately.

to the original grouping in the segregation by motion offset display $(M=691.6 \mathrm{msec})$ relative to the segregation by continuous motion display $(M=656.3 \mathrm{msec})[t(9)=$ $5.08, p<.005]$. A similar pattern of results was not seen for the new object condition $[t(9)=0.61, p=$ n.s.].

Of note, in the segregation by continuous motion display, the original grouping was stationary at the time of target identification, whereas in the segregation by motion offset display, the original grouping was still in motion at the time of target identification. It follows that the difference in RTs seen across the two display types might be related to a general difficulty in identifying targets that are moving relative to ones that are static. If this is true, then one might ask why a similar difference was not seen for the newly segregated item conditions in the present experiment. In particular, if moving objects are more difficult to identify than stationary ones (as we have just sug- 
gested), then target identification should have been slower when the target appeared in the newly segregated item in the segregation by continuous motion display (because the target was moving in that display) relative to the segregation by motion offset display (because the target was stationary in that display). One reason such a difference was not observed may be due to the fact that attention is presumed to have been captured by the newly segregated item in both conditions. As Yeshurun and Carrasco $(1998,2000)$ have shown, one consequence of allocating attention to a location is an enhancement of perceptual sensitivity at the attended location. The increased sensitivity at the attended location may explain the absence of a detrimental effect of target motion in the segregation by continuous motion condition.

\section{EXPERIMENT 3}

In Experiments 1 and 2, the subjects were faster to respond when the target appeared in a newly segregated item than when it appeared in the original grouping. Whereas this pattern of results is consistent with attentional capture by the newly segregated item, we sought to provide additional evidence of attentional capture using a set size manipulation. If attention is captured by the newly segregated item, then one would expect for target search to be much more efficient and load insensitive when the target appears in the newly segregated item than when it appears in the original grouping (Yantis \& Jonides, 1990).

\section{Method}

Subjects. Fifteen students drawn from the same population as studied earlier participated. None had served previously.

Apparatus, Procedure, and Design. This experiment was similar to Experiment 2, with differences noted here. On half of the trials, the initial grouping consisted of four placeholders. On the other half, there were eight placeholders. On each trial, one of the elements was segregated by either motion offset or continuous motion. The placeholders were changed to reveal the search display coincident with the movement transition. The target was equally likely to appear in each of the elements of the search display (set size of four, $25 \%$; set size of eight, $12.5 \%$ ). Following 20 practice trials, the subjects served in 384 experimental trials. Trial types were randomly mixed.

\section{Results and Discussion}

Mean RTs from each condition are shown in Figure 4. The data were analyzed using a 2 (display; segregation by motion offset vs. segregation by continuous motion) $\times 2$ (target; segregated item vs. original grouping) $\times 2$ (set size; four items vs. eight items) ANOVA. A main effect of target was observed, with the subjects responding faster when the target appeared in the segregated item than when it appeared in the original grouping $[F(1,14)=203.19$, $p<.001]$. The subjects were also slower overall when the display size was eight than when it was four $[F(1,14)=$ 98.06, $p<.001]$.

Most important, an interaction between target and set size was evident. The search slope when the target appeared in the original group ( $30.5 \mathrm{msec}$ per item) was significantly steeper than when it appeared in the newly

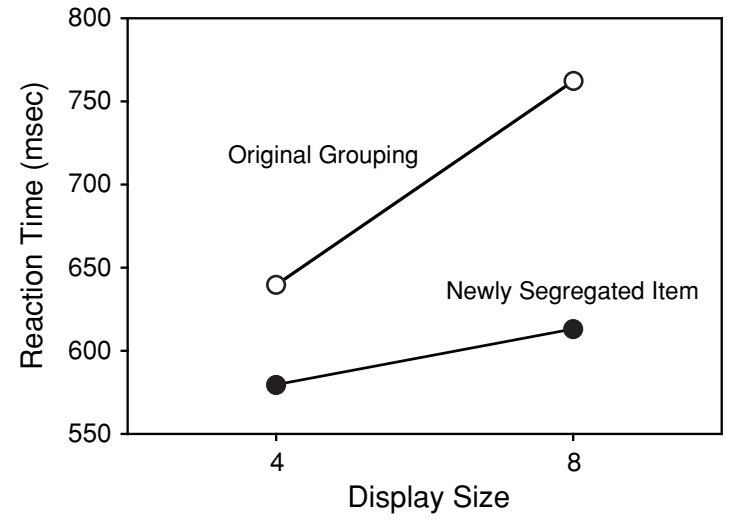

Figure 4. Mean reaction times for target identification in Experiment 3 , shown separately for each display size (4 items and 8 items) and each target type (newly segregated item or original grouping). Given that there was no main effect of display type (segregation $\times$ motion offset and segregation $\times$ continuous motion) and display type did not interact with any of the other experimental variables, data were collapsed over display type for presentation purposes.

segregated item ( $8.2 \mathrm{msec}$ per item). These slopes are in the range generally believed to represent inefficient and "quite efficient" search, respectively (Wolfe, 1998). This pattern of results is consistent with attentional capture by the newly segregated item. No other main effect or interaction reached statistical significant $\left[F_{\mathrm{s}}(1,14)<2.5\right.$, $p>.1$, in all instances].

Error rates were less than 5\% in all conditions and did not depend on target or display type, nor was there an interaction $\left[F_{\mathrm{s}}(1,14)<1\right]$.

Taken together with the results from Experiments 1 and 2, the present findings provide strong evidence that a newly segregated item does indeed capture attention in an automatic, bottom-up manner. Even though the location of the target was uncorrelated with the segregation status of each display item, visual search was significantly more efficient when the target appeared in a newly segregated item than when it appeared in a member of the original grouping.

\section{EXPERIMENT 4}

Although all evidence presented thus far is consistent with bottom-up attentional capture by a newly segregated item, an alternate explanation for our pattern of results exists. Namely, the subjects may be adopting a top-down attentional set for segregation. ${ }^{2}$ Indeed, given that there is some uncertainty as to the precise timing of the target presentation, and given that target presentation always coincides with the movement transition (i.e., segregation by motion offset or continuous motion), the subjects might have been attending to the segregation in order to ascertain whether or not the target display was present. To rule out this possibility, we conducted an additional experiment.

In Experiment 4, all uncertainty regarding the timing of the target display presentation was removed. By doing 
so, we eliminated any strategic benefit of attending to the movement transition and thus removed a major motivation for adopting a top-down set for segregation.

\section{Method}

Subjects. Fifteen students drawn from the same population as studied earlier participated. None had served previously.

Apparatus, Procedure, and Design. This experiment was similar to Experiment 2, with one difference: Throughout trial presentation, the horizontal location of target presentation was clearly marked by two arrows directly above and below the movement path of the stimulus grouping. When the grouping came into vertical alignment with the arrows, the target display was presented and the movement transition occurred. The sequence of events on a segregation by motion offset trial is shown in Figure 5 .

The target was equally likely to appear in each of the five items. The subjects were instructed to identify the target as quickly as possible. Following 20 practice trials, the subjects served in 360 experimental trials. Trial types were randomly mixed.

\section{Results and Discussion}

Data from 1 subject was excluded from further analysis due to excessive errors $(20 \%)$.

Mean RTs from each condition are shown in the right pairs of bars in Figure 2. The data were analyzed using a 2 (target; newly segregated item vs. original grouping) $\times$ 2 (display; segregation by motion offset vs. segregation by continuous motion) ANOVA. A main effect of target type was evident $[F(1,13)=31.38, p<.001]$. Similar to the previous experiments, the subjects responded faster when the target appeared in the newly segregated item than when it appeared in the original grouping. There was no main effect of segregation type $[F(1,13)<1.0]$, nor was there a significant interaction $[F(1,13)=4.18, p>$ $.05]$.
Error rates were less than 5\% in all conditions and did not depend on target or display type, nor was there an interaction $\left[F_{\mathrm{S}}(1,13)<2.3\right]$.

The present results confirm that a newly segregated item continues to capture attention even after all uncertainty as to the timing of the presentation of the target display has been removed. In fact, the RT benefit observed for the newly segregated item in Experiment 4 (72 $\mathrm{msec})$ was comparable to that observed in Experiment 2 (70 $\mathrm{msec})$. If earlier results were due to a top-down attentional set for segregation, however, then one would expect the size of this attentional benefit to be substantially reduced in Experiment 4 relative to that in Experiment 2 (which it was not).

\section{EXPERIMENT 5}

In Experiments 1-4, the finding that the subjects were faster to respond when the target appeared in a newly segregated item than when it appeared in the original grouping was taken as evidence of attentional capture. Although this pattern of results is indeed consistent with attentional capture by the newly segregated item, an alternative explanation still exists. Specifically, it may be the case that subjects are simply slower to respond to a target when it is grouped with distractor stimuli than when it is not (e.g., Banks \& Prinzmetal, 1976). Or, in a similar manner, lateral masking may adversely affect target identification when it is close to other stimuli (Cohen \& Ivry, 1991).

In Experiment 5, we directly compared the RT advantage afforded to a newly segregated item with that of an item that had not recently been segregated but remained spatially separate from a grouping of items. If grouping or lateral masking accounted for our earlier results, then

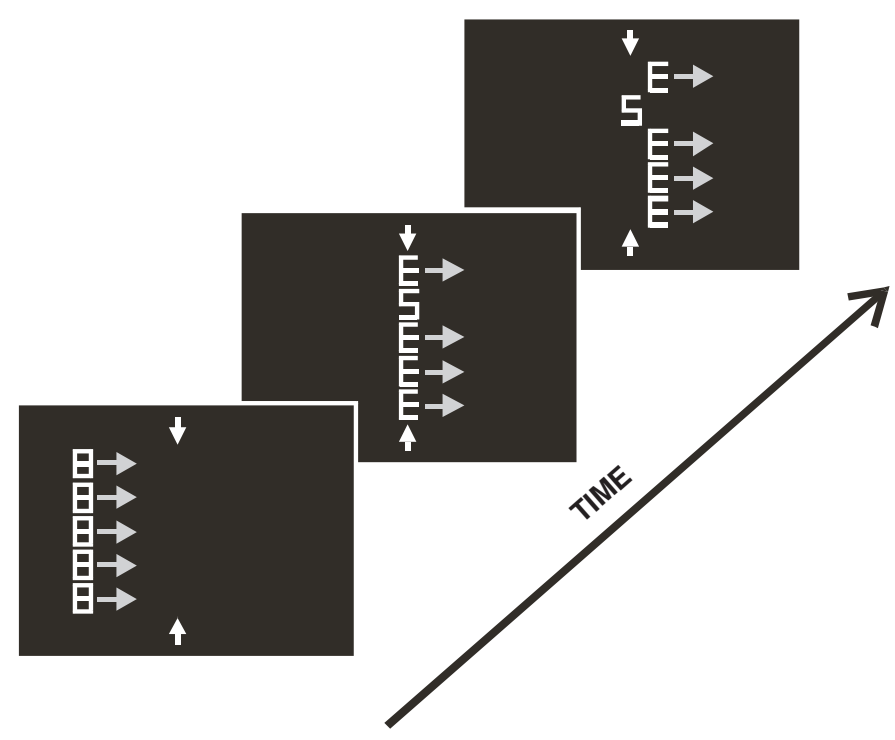

Figure 5. Sequence of events on a segregation by motion offset trial in Experiment 4. (The gray horizontal arrows are pictured for illustrative purposes only and represent the movement vectors of the display items.) 
there should be no difference between the two conditions; however, if attention is truly captured by the "newness" of the newly segregated item, then the attentional benefit would be greatest at the time of segregation.

\section{Method}

Subjects. Eleven students drawn from the same population as studied earlier participated. None had served previously.

Apparatus, Procedure, and Design. On two thirds of the trials, trial presentation was identical to that described in Experiment 2. For these trials, a grouping of five placeholders moved across the display from left to right. Following a random delay of 1,667-5,000 msec, a movement transition (segregation by motion offset or continuous motion) occurred concurrent with target display presentation.

On the remaining one third of the trials (the static segregation condition), there was no initial motion nor any later motion transition presented. The initial display consisted of a row of four vertically aligned placeholders located within $5^{\circ}$ of the center of the display. A single placeholder was located $0.75^{\circ}$ to the left or right of the larger grouping and was horizontally aligned with an unoccupied space in the larger grouping. In other words, the position of the items in the static segregation condition was designed to mimic the spatial arrangement observed in the other segregation conditions approximately $550 \mathrm{msec}$ after target presentation - a time past when perceptual processing of the target display was likely completed in most instances (the average RT from Experiment 2 was $639 \mathrm{msec}$ ). Following a random delay of $1,667-5,000 \mathrm{msec}$, the placeholders were changed to reveal the search display.

Across all three segregation conditions, the target was equally likely to appear in each of the five items, and the subjects were instructed to identify the target as quickly as possible. Following 20 practice trials, the subjects served in 360 experimental trials. Trial types were randomly mixed.

\section{Results and Discussion}

Mean RTs from each condition are shown in Figure 6. The data were analyzed using a 2 (target; segregated item vs. original grouping) $\times 3$ (display; segregation by motion offset vs. segregation by continuous motion vs. static segregation) ANOVA. The main effect of display approached significance, with the subjects responding fastest on segregation by continuous motion trials $(M=$ $627 \mathrm{msec}$ ) and slightly slower on the segregation by motion offset trials $(M=634 \mathrm{msec})$ and static segregation trials $(M=658 \mathrm{msec})[F(2,20)=3.39, p>.05]$.

Overall, the subjects were faster to respond when the target was in the newly segregated item relative to the existing group $[F(1,10)=17.76, p<.005]$. This effect, however, was clearly driven by performance in the segregation by motion offset and segregation by continuous motion conditions (existing group RT minus newly segregated item RT $=83$ and $60 \mathrm{msec}$, respectively). There was not a similar benefit for the segregated item in the static segregation condition as evidenced by the presence of an interaction between target and display $[F(2,20)=9.16$, $p<.005]$. In fact, the reverse was true for static segregation trials: the subjects were $14 \mathrm{msec}$ faster to respond when the target was in the existing group than when the target was in the segregated item. This pattern of results is consistent with attentional capture by the newly segregated item.

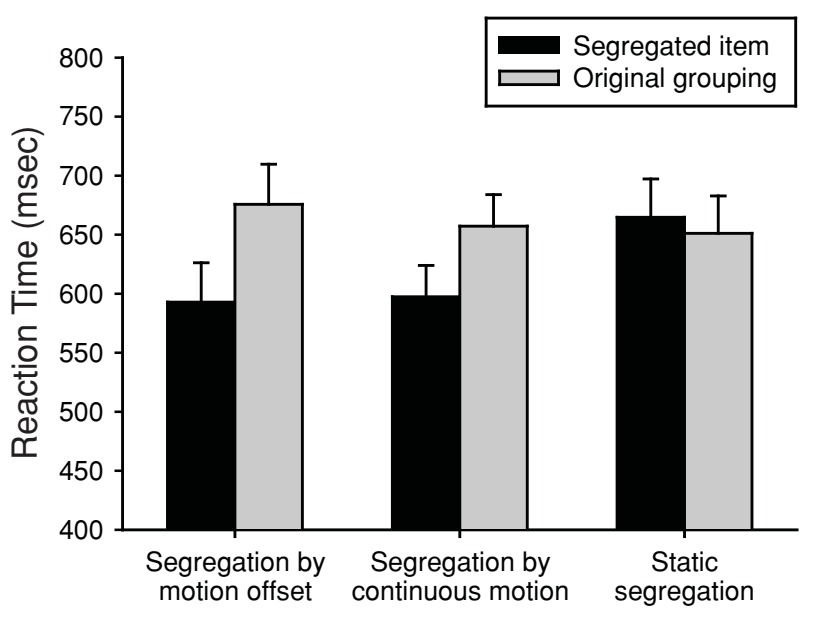

Figure 6. Mean reaction times for target identification in Experiment 5 , shown separately for each display type (segregation by motion offset, segregation by continuous motion, or static segregation) and each target type (segregated item or original grouping). Error bars show the standard error of the mean.

Error rates were less than $4 \%$ in all conditions and did not depend on target or display type, nor was there an interaction ( $p \mathrm{~s}>.2$, in all instances).

The results from Experiment 5 rule out an alternative explanation for our findings from Experiments 1-4. That is, it was possible that the slower RTs to items in the original grouping may have been entirely due to an overall interference or lateral masking effect caused by the target being grouped with distractors at the time of target identification (which was never the case for the newly segregated item). This explanation, however, cannot account for the attentional advantage seen in Experiment 5 for the segregated item when it was "new" relative to when it was "old."

\section{GENERAL DISCUSSION}

In the present experiments, we have shown an advantage to identify targets that are contained in preexisting elements that have recently become segregated from a group. The advantage was greatest at the time of segregation, and it was realized when the element became segregated either by moving away from the group or by the group moving away from the element. Importantly, the result occurred in scenes where the only change in motion was an offset of movement, and in which the segregated element did not undergo any change in its motion at all (Experiment 2). Thus, these results show that segregation is sufficient to attract attention in the absence of motion cues.

In our displays, all of the elements were clearly visible from the beginning of the trial, so all of the items could technically be regarded as "old." However, by becoming segregated from the others in the group, one item was made to stand out - and we think this item was seen by the perceptual system as a "new" object and thus captured attention. 
If one conceives of the internal representations that we have of the objects in a scene as being contained in "object files" (Kahneman, Treisman, \& Gibbs, 1992), then the high attentional priority assigned to new objects might be regarded as something that naturally occurs when a new object file is created (Yantis \& Hillstrom, 1994). Such an event would be expected any time a new object appeared, regardless of the nature of the display change that caused the new object to become apparent.

Within this context, the newly segregated item in our experiments was no longer part of a group, and, hence, it now required its own internal representation (e.g., "object file"). The need to create such a representation apparently attracted attention. Thus, we conceive of our results as a special case of attentional capture by a new perceptual object (cf. Yantis \& Hillstrom, 1994).

Our conclusions are similar in some respects to those from a recent study by Rauschenberger (2003b). He examined the attentional consequences of changes to features of preexisting objects when subjects were set to expect a new object to appear. He showed that objects that undergo some, but not all, types of changes do indeed have an impact on attention that is similar to the impact of a new object. Likewise, we have shown that a new segmentation of elements in a scene may cause some existing elements be treated as a new object. Recent work by Raymond (2003) also has helped to distinguish the attentional consequences of a featural change to an existing object from the consequences of the appearance of an entirely new object. Eventually, a complete understanding of these effects may depend on, or lead to, a better understanding of precisely what it is that constitutes a new object (see Scholl, Pylyshyn, \& Feldman, 2001, for a discussion of this issue).

Our results are also broadly consistent with recent work that further bolsters the importance of objects in general for attentional selection. There is considerable evidence that attention operates upon representations of the objects in a scene (e.g., Abrams \& Law, 2000; Duncan, 1984; Egly, Driver, \& Rafal, 1994; He, Fan, Zhou, \& Chen, 2004; Law \& Abrams, 2002). Additionally, recent results have shown that the appearance of a new perceptual object is more effective at capturing attention than other transient events, such as object disappearance (Samuel \& Weiner, 2001) and luminance changes (Enns, Austen, Di Lollo, Rauschenberger, \& Yantis, 2001). The present results extend these earlier findings by showing that an object might be considered "new" even when it (1) is not represented by a new visual stimulus and (2) has not undergone significant changes to any of its featural attributes. These findings attest to the importance of objects in the establishment of attentional priorities since, as shown here, even an "old" object may be seen as "new" when the interpretation of a scene changes.

\section{REFERENCES}

Abrams, R. A., \& Christ, S. E. (2003). Motion onset captures attention. Psychological Science, 14, 427-432.

Abrams, R. A., \& Christ, S. E. (2004a, May). Automatic capture of at- tention by the onset of motion. Poster presented at the annual meeting of the Vision Sciences Society, Sarasota, FL.

Abrams, R. A., \& Christ, S. E. (2004b). Capture of attention by motion onset in random dot displays. Manuscript submitted for publication.

Abrams, R. A., \& Christ, S. E. (2005). Onset but not offset of irrelevant motion disrupts inhibition of return. Perception \& Psychophysics, 67, 1460-1467.

Abrams, R. A., \& LAW, M. B. (2000). Object-based visual attention with endogenous orienting. Perception \& Psychophysics, 62, 818-833.

BanKs, W. P., \& PRInZMETAL, W. (1976). Configurational effects in visual information processing. Perception \& Psychophysics, 19, 361-367.

Cohen, A., \& Ivry, R. (1991). Density effects in conjunction search: Evidence for a coarse location mechanism of feature integration. Journal of Experimental Psychology: Human Perception \& Performance, 17, 891-901.

Duncan, J. (1984). Selective attention and the organization of visual information. Journal of Experimental Psychology: General, 113, 501517.

Egly, R., Driver, J., \& Rafal, R. D. (1994). Shifting visual attention between objects and locations: Evidence from normal and parietal lesion subjects. Journal of Experimental Psychology: General, 123, 161-177.

Enns, J. T., Austen, E. L., Di Lollo, V., Rauschenberger, R., \& YANTIS, S. (2001). New objects dominate luminance transients in setting attentional priority. Journal of Experimental Psychology: Human Perception \& Performance, 27, 1287-1302.

Gellatly, A., Cole, G., \& Blurton, A. (1999). Do equiluminant object onsets capture visual attention? Journal of Experimental Psychology: Human Perception \& Performance, 25, 1609-1624.

He, X., FAn, S., Zhou, K., \& Chen, L. (2004). Cue validity and objectbased attention. Journal of Cognitive Neuroscience, 16, 1085-1097.

Hillstrom, A. P., \& Yantis, S. (1994). Visual motion and attentional capture. Perception \& Psychophysics, 55, 399-411.

Kahneman, D., Treisman, A., \& GibBs, B. J. (1992). The reviewing of object files: Object-specific integration of information. Cognitive Psychology, 24, 175-219.

Law, M. B., \& Abrams, R. A. (2002). Object-based selection within and beyond the focus of spatial attention. Perception \& Psychophysics, 64, 1017-1027.

OonK, H. M., \& Abrams, R. A. (1998). New perceptual objects that capture attention produce inhibition of return. Psychonomic Bulletin \& Review, 5, 510-515.

RAuschenberger, R. (2003a). Attentional capture by auto- and allocues. Psychonomic Bulletin \& Review, 10, 814-842.

RAUSCHENBERGER, R. (2003b). When something old becomes something new: Spatiotemporal object continuity and attentional capture. Journal of Experimental Psychology: Human Perception \& Performance, 29, 600-615.

Rauschenberger, R., \& Yantis, S. (2001). Attentional capture by globally defined objects. Perception \& Psychophysics, 63, 1250-1261.

RAYMOND, J. E. (2003). New objects, not new features, trigger the attentional blink. Psychological Science, 14, 54-59.

Samuel, A. G., \& Weiner, S. K. (2001). Attentional consequences of object appearance and disappearance. Journal of Experimental Psychology: Human Perception \& Performance, 27, 1433-1451.

Scholl, B. J., Pylyshyn, Z. W., \& Feldman, J. (2001). What is a visual object? Evidence from target merging in multiple object tracking. Cognition, 80, 159-177.

WoLfe, J. (1998). Visual search. In H. Pashler (Ed.), Attention (pp. 1373). London: University College London Press.

YanTis, S., \& Hillstrom, A. P. (1994). Stimulus-driven attentional capture: Evidence from equiluminant visual objects. Journal of Experimental Psychology: Human Perception \& Performance, 20, 95-107.

Yantis, S., \& Jonides, J. (1984). Abrupt visual onsets and selective attention: Evidence from visual search. Journal of Experimental Psychology: Human Perception \& Performance, 10, 601-621.

Yantis, S., \& Jonides, J. (1990). Abrupt visual onsets and selective attention: Voluntary versus automatic allocation. Journal of Experimental Psychology: Human Perception \& Performance, 16, 121-134.

YeshuRUn, Y., \& CARRASCO, M. (1998). Attention improves or impairs visual performance by enhancing spatial resolution. Nature, 396, 7275 . 
Yeshurun, Y., \& Carrasco, M. (2000). The locus of attentional effects in texture segmentation. Nature Neuroscience, 3, 622-627.

\section{NOTES}

1. The topmost and bottommost items are adjacent to a single member of the grouping whereas the remaining items are each adjacent to two members of the grouping. This raised the concern that the strength of the segregation manipulation might differ for these two items relative to the other items in the display. As such, the topmost and bottommost items never served as the segregated item. One might wonder as to whether or not this imposed constraint may have influenced subjects' attentional treatment of these items (they continued to serve as potential target lo- cations). On the basis of the RT data, the answer is "no": The subjects were equally fast to respond when the target appeared in the topmost or bottommost item as when it appeared in another member of the original grouping ( $p \mathrm{~s}>.1$, in all instances).

2. One could argue that a top-down attentional set for segregation is not sufficient to explain our results. Specifically, since the segregation itself involves both the newly segregated item and the original grouping, there is no reason to believe that a set for segregation would unequally bias RTs for one more so than the other.

(Manuscript received May 5, 2003; revision accepted for publication May 9, 2005.) 